Supporting Information

\title{
Heterodinuclear Bridged Borylene Complexes
}

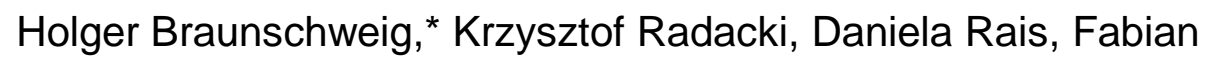

Seeler, and Katharina Uttinger

Institut für Anorganische Chemie, Bayerische Julius-Maximilians-

Universität Würzburg, Am Hubland, D-97074 Würzburg, Germany 


\section{General considerations}

All manipulations were conducted either under an atmosphere of dry argon or in vacuo using standard Schlenk line or glove-box techniques. Solvents (toluene, benzene and hexane) were purified by distillation from appropriate drying agents (sodium and sodium wire) under dry argon, immediately prior to use. Deuterated solvents $\left(\mathrm{C}_{6} \mathrm{D}_{6}\right.$ and toluene$d_{8}$ ) were degassed by three freeze-pump-thaw cycles and stored over molecular sieves in the glove-box. IR spectra for compounds were recorded as toluene solutions between $\mathrm{KBr}$ plates on a Bruker Vector $22 \mathrm{FT}-\mathrm{IR}$-spectrometer. NMR spectra were recorded on Bruker AMX 400 and Bruker Avance 500 NMR spectrometers. Variable temperature NMR experiments were performed on a Bruker Varian 200 NMR spectrometer. ${ }^{1} \mathrm{H}$ and ${ }^{13} \mathrm{C}\left\{{ }^{1} \mathrm{H}\right\}$ NMR spectra were referenced to external TMS via the residual protio solvent $\left({ }^{1} \mathrm{H}\right)$ or the solvent itself $\left({ }^{13} \mathrm{C}\right) .{ }^{11} \mathrm{~B}\left\{{ }^{1} \mathrm{H}\right\}$ and ${ }^{31} \mathrm{P}\left\{{ }^{1} \mathrm{H}\right\}$ NMR spectra were referenced to external $\mathrm{BF}_{3} \mathrm{OEt}_{2}$ and $85 \% \mathrm{H}_{3} \mathrm{PO}_{4}$, respectively. Microanalyses for $\mathrm{C}, \mathrm{H}$ and $\mathrm{N}$ were performed by Mr C. P. Kneis (University of Würzburg) on a Leco CHNS-932 instrument. For cyclopentadienyl carbon atom labeling in $\mathbf{3}$, see Figure 1 in text.

\section{Synthetic procedures}

$\left[\left(\eta^{5}-\mathrm{C}_{5} \mathrm{Me}_{5}\right)(\mathrm{CO}) \mathrm{Fe}(\mu-\mathrm{CO})(\mu-\mathrm{BFc}) \mathrm{Pd}(\mathrm{Br})\left(\mathrm{PCy}_{3}\right)\right](3)$ : A solution of $\left[\mathrm{Pd}\left(\mathrm{PCy}_{3}\right)_{2}\right](2)$ $(0.064 \mathrm{~g}, \quad 0.096 \mathrm{mmol})$ in $\mathrm{C}_{6} \mathrm{D}_{6}(0.5 \mathrm{~mL})$ was added to a solution of $\left[\left(\eta^{5}-\right.\right.$ $\left.\left.\mathrm{C}_{5} \mathrm{Me}_{5}\right)(\mathrm{OC})_{2} \mathrm{Fe}\{\mathrm{B}(\mathrm{Fc}) \mathrm{Br}\}\right](\mathbf{1})(0.050 \mathrm{~g}, 0.096 \mathrm{mmol})$ in $\mathrm{C}_{6} \mathrm{D}_{6}(0.5 \mathrm{~mL})$. The initial dark red solution gradually turned bright red over the course of four days. Hexane ( $c a 3 \mathrm{~mL}$ ) was carefully layered on the solution and the mixture was cooled to $-30{ }^{\circ} \mathrm{C}$. After 2 days orange-red crystals of $\mathbf{3}$ were isolated and dried in vacuo (yield $0.073 \mathrm{~g}, 84 \%$ ). ${ }^{1} \mathrm{H}$ NMR 
(500 MHz, toluene- $\left.d_{8}, 25^{\circ} \mathrm{C}, \mathrm{TMS}\right): \delta=6.16\left[\mathrm{br} \mathrm{s}, 1 \mathrm{H}, \mathrm{C}(14)-H, \mathrm{BC}_{5} H_{4}\right], 4.40-4.36[\mathrm{br}$ m, 3H, C(11)-H, C(12)-H, C(13)-H, $\left.\mathrm{BC}_{5} H_{4}\right], 4.25$ (s, 5H, $\left.\mathrm{C}_{5} H_{5}\right), 2.64$ (m, 3H, $\left.\mathrm{C}_{1} H, \mathrm{Cy}\right)$, 2.17-1.24 (m, 30H, Cy), 1.76 (s, $\left.15 \mathrm{H}, \mathrm{C}_{5} M e_{5}\right) ;{ }^{13} \mathrm{C}$ NMR (126 MHz, toluene- $\left.d_{8}, 25{ }^{\circ} \mathrm{C}\right): \delta$ $=251.2(\mathrm{~s}, \mathrm{CO}), 214.0(\mathrm{~s}, \mathrm{CO}), 97.1\left(\mathrm{~s}, C_{5} \mathrm{Me}_{5}\right), 80.0\left(\mathrm{br} \mathrm{s}, \mathrm{B} C_{4} \mathrm{H}_{5}\right), 76.8\left(\mathrm{br} \mathrm{s}, \mathrm{B} C_{4} \mathrm{H}_{5}\right)$, $74.2\left(\mathrm{~s}, \mathrm{~B} C_{4} \mathrm{H}_{5}\right), 69.3\left(\mathrm{~s}, C_{5} \mathrm{H}_{5}\right), 31.9\left(\mathrm{~d},{ }^{1} \mathrm{~J}_{\mathrm{C}-\mathrm{P}}=10 \mathrm{~Hz}, \mathrm{C}_{1}, \mathrm{Cy}\right), 30.8$ (br s, $\left.\mathrm{C}_{3,5}, \mathrm{Cy}\right), 28.1$ $\left(\mathrm{d},{ }^{2} \mathrm{~J}_{\mathrm{C}-\mathrm{P}}=10 \mathrm{~Hz}, \mathrm{C}_{2,6}, \mathrm{Cy}\right), 27.1\left(\mathrm{~s}, \mathrm{C}_{4}, \mathrm{Cy}\right), 10.3\left(\mathrm{~s}, \mathrm{C}_{5} M e_{5}\right) ;{ }^{31} \mathrm{P} \mathrm{NMR}(202 \mathrm{MHz}$, toluene- $\left.d_{8}, 25{ }^{\circ} \mathrm{C}\right): \delta=23.9$ (br s); ${ }^{11} \mathrm{~B}\left\{{ }^{1} \mathrm{H}\right\}$ NMR $\left(160 \mathrm{MHz}\right.$, toluene- $\left.d_{8}, 25{ }^{\circ} \mathrm{C}\right): \delta=136$ (br s, $\omega_{1 / 2}=1780 \mathrm{~Hz}$ ). IR (toluene): 1952, $1794 \mathrm{~cm}^{-1}, v(\mathrm{C}=\mathrm{O})$. Elemental Analyses: calcd for $\mathrm{C}_{40} \mathrm{H}_{57} \mathrm{BBrFe}_{2} \mathrm{O}_{2} \mathrm{PPd} \cdot \mathrm{C}_{7} \mathrm{H}_{8}$ : C 56.35, H 6.54; found: C 55.67, H 6.30.

$\left[\left(\eta^{5}-\mathrm{C}_{5} \mathrm{Me}_{5}\right)(\mathrm{CO}) \mathrm{Fe}(\mu-\mathrm{CO})(\mu-\mathrm{BBr}) \mathrm{Pd}(\mathrm{Br})\left(\mathrm{PCy}_{3}\right)\right](\mathbf{5}):$ A solution of $\left[\mathrm{Pd}\left(\mathrm{PCy}_{3}\right)_{2}\right](\mathbf{2})$ $(0.080 \mathrm{~g}, 0.12 \mathrm{mmol})$ in hexane $(1.5 \mathrm{~mL})$ was added to a solution of $\left[\left(\eta^{5}-\right.\right.$ $\left.\left.\mathrm{C}_{5} \mathrm{Me}_{5}\right)(\mathrm{OC})_{2} \mathrm{Fe}\left(\mathrm{BBr}_{2}\right)\right](4)(0.050 \mathrm{~g}, 0.12 \mathrm{mmol})$ in hexane (1.5 mL). After stirring for 5 min, the solution turned dark red. The mixture was cooled to $-30{ }^{\circ} \mathrm{C}$, yielding a yellow precipitate of $\mathbf{5}$ over the course of 2 days. This was isolated, washed with hexane and dried in vacuo (yield $0.062 \mathrm{~g}, 64 \%$ ). ${ }^{1} \mathrm{H}$ NMR (400 MHz, $\mathrm{C}_{6} \mathrm{D}_{6}, 25{ }^{\circ} \mathrm{C}$ ): $\delta=2.90-2.75$ (m, 3H, $\left.\mathrm{C}_{1} H, \mathrm{Cy}\right), 2.18-1.10$ (m, 30H, Cy), 1.63 (s, 15H, $\left.\mathrm{C}_{5} M e_{5}\right) ;{ }^{13} \mathrm{C}$ NMR (101 MHz, $\left.\mathrm{C}_{6} \mathrm{D}_{6}, 25^{\circ} \mathrm{C}\right): \delta=98.3\left(\mathrm{~s}, C_{5} \mathrm{Me}_{5}\right), 34.1\left(\mathrm{~d},{ }^{1} \mathrm{~J}_{\mathrm{C}-\mathrm{P}}=18 \mathrm{~Hz}, \mathrm{C}_{1}, \mathrm{Cy}\right), 31.0\left(\mathrm{~s}, \mathrm{C}_{3,5}, \mathrm{Cy}\right), 27.9$ $\left(\mathrm{d},{ }^{2} \mathrm{~J}_{\mathrm{C}-\mathrm{P}}=11 \mathrm{~Hz}, \mathrm{C}_{2,6}, \mathrm{Cy}\right), 26.9\left(\mathrm{~s}, \mathrm{C}_{4}, \mathrm{Cy}\right), 10.2\left(\mathrm{~s}, \mathrm{C}_{5} M e_{5}\right), \mathrm{CO}$ resonances were not detected. Due to the low solubility of $\mathbf{5}$ in $\mathrm{C}_{6} \mathrm{D}_{6}$, only a relatively small amount of the compound could be used for the acquisition of the spectrum; ${ }^{31} \mathrm{P}$ NMR $\left(162 \mathrm{MHz}, \mathrm{C}_{6} \mathrm{D}_{6}\right.$, $\left.25^{\circ} \mathrm{C}\right): \delta=45.6(\mathrm{~s}) ;{ }^{11} \mathrm{~B}\left\{{ }^{1} \mathrm{H}\right\} \mathrm{NMR}\left(64 \mathrm{MHz}, \mathrm{C}_{6} \mathrm{D}_{6}, 25^{\circ} \mathrm{C}\right): \delta=118\left(\mathrm{br} \mathrm{s}, \omega_{1 / 2}=526\right.$ Hz). IR (toluene): 1939, $1802 \mathrm{~cm}^{-1}, v(\mathrm{C}=\mathrm{O})$. Elemental Analyses: calcd for $\mathrm{C}_{30} \mathrm{H}_{48} \mathrm{BBr}_{2} \mathrm{FeO}_{2} \mathrm{PPd}: \mathrm{C} 44.79$, H 6.01; found: C 44.77, H 5.94. 